\title{
La misura della disomogeneità di magnetizzazione delle rocce $(*)$
}

(The measure of inhomogeneity of rock-magnetization).

\author{
R. ChaLdenes
}

Ricevuto il 20 Giugno 1967

\begin{abstract}
Riassunto. - Nella presente nota viene mostrato l'uso del magnetometro, precedentemente descritto dall'A., nella misura della disomogeneita di magnetizzazione di un campione di roccia.

A tale scopo si studia anzitutto la forma del segnale generato in questo magnetometro da un dipolo decentrato; poi si schematizza un generico campione di roccia come un campione magnetizzato uniformemente in una certa direzione avente in più un dipolo decentrato non necessariamente parallelo alla sopradetta direzione.

Con questa schematizzazione $\mathrm{l}^{\prime} \mathrm{A}$. introduce una grandezza, chiamata indice di disomogeneità di magnetizzazione, che può dare un'idea delle eventuali disomogeneità di magnetizzazione presentate dal campione.
\end{abstract}

SUmmaRY. - - The use of the magnetometer, previous described by the $A$, in the measure of the magnetization inhomogeneity of a rock sample is shown in the present paper.

At the first it is studied the signal generated in this magnetometer by an off-centre dipole; after it is proposed to reduce a rock sample to a sample uniformly magnetized in a direction with an off-centre dipole no necessary parallel to that direction.

With this scheme the A. introduces a measure, that it named the index of the magnetization inhomogeneity: of course this index can do an idea of the eventual inhomogeneity of the rock sample.

In una precedente nota (1) ho descritto un nuovo tipo di magnetometro per le misure magnetiche sulle rocee, destinato al rilevamento

(*) Questa nota ò stata presentata al XVI Convegno iell'A. G. I. Napoli, 22-24 Maggio 1967. 
della direzione dell'intensità di magnetizzazione. In essa è riportata la relativa teoria da me elaborata studiando in particolare la forma del segnale generato da un campione di roccia supposta magnetizzata uniformemente in una generica direzione.

Con questo magnetometro, realizzato nell'Istituto Nazionale di Grofisica e già in uso da parecchi mesi, ho osservato che la forma del segnale spesso era analoga a quella teorica, trovata e descritta nella nota citata, ma talvolta, se ne discostava moltissimo. Un primo esame del fenomeno mi fece suppore che tale diverso comportamento era doruto essenzialmente al fatto che non tutti i campioni prelevati presentavano una magnetizzazione uniforme. Pertanto nella presente nota studierò la forma del segnale che si ottiene con questo magnetometro esaminando una roceia presentante una magnetizzazione disuniforme $\left({ }^{2}\right)$.

Premetto anzitutto le notazioni, in gran parte gia adoperate nella nota citata:

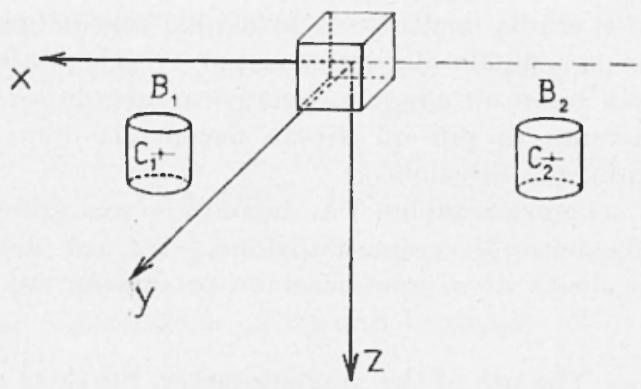

Fig. 1

$0, x, y, z$ : terna ortogonale fissa con l'origine nel centro geometrico del campione nella sua posizione di equilibrio, l'asse $x$ parallelo alla congiungente i centri $C_{1}$ e $C_{2}$ delle due bobine $B_{1}$ e $B_{2}$. l'asse $y$ giacente sul piano orizzontale a l'asse $z$ lungo la verticale (Fig. 1),

$\Omega, \xi, \eta, \zeta$ : terna ortogronale solidale al campione e con l'origine $\Omega$ coincidente con $O$ nella sua posizione di equlibrio,

$r \quad$ : raggio delle due bobine,

$l \quad:$ lunghezza del pendolo,

a : angolo formato dall'asse del pendolo in un generico istante con la verticale (Figr. 2), 
$\alpha_{0} \quad$ : valore massimo (in modulo) di $\alpha$, $\omega=l \overline{g / l}$ : pulsazione propria del pendolo,

$\vec{m} \quad$ : momento magnetico del campione in esame,



Fig. 2

$\psi \quad$ : angolo formato dal vettore $\vec{m}$ con l'asse $\zeta$ (Fig. 3),

$\chi \quad$ : angolo formato dalla componente orizzontale $m_{0}$ di $\vec{m}$ con l'asse $\eta$ (Fig. 3),

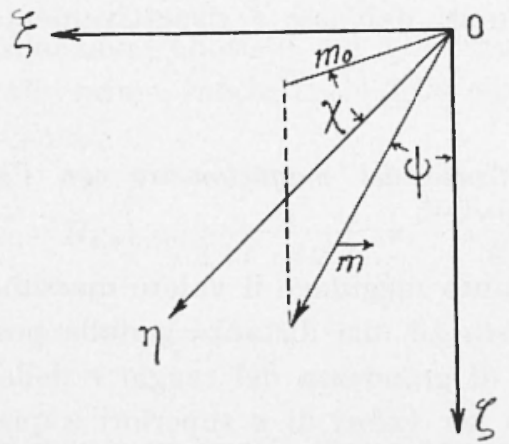

Fig. 3

$H_{1}$ e $H_{2}$ : valore assunto dalla componente verticale del campo magnetico generato dal campione nei due centri $C_{1}$ e $C_{2}$ rispettivamente,

$a, o, h$ : coordinate di $C_{1}$ rispetto alla terma $O, x, y, z$, 
$a, 0, h$ : coordinate di $C_{2}$ rispetto alla terma $O, x, y, z$,

$o, y, z \quad$ : coordinate di $\Omega$ rispetto alla terna $O, x, y, z$,

$\lambda_{x}, \lambda_{y}, \lambda_{z}$ : angoli formati dall'asse $\zeta$ rispettivamente con gli assi $x, y, z$ (Fig. 4),

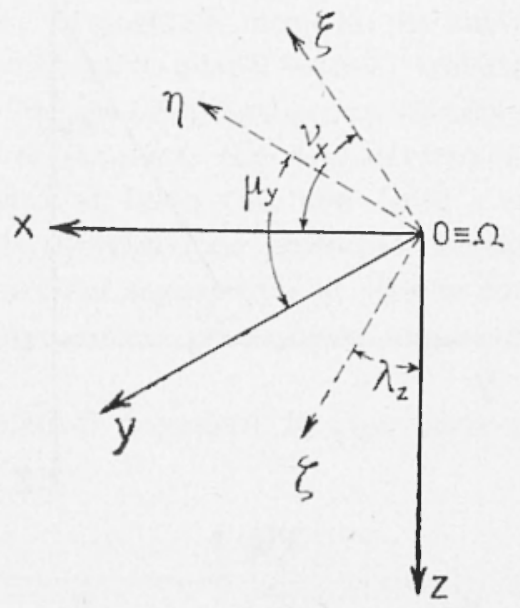

Fig. 4

$\mu_{x}, \mu_{y}, \mu_{z}$ : angoli formati dall'asse $\eta$ rispettivamente con gli assi $x, y, z$ (Fig. 4),

$\nu_{x}, v_{y}, \nu_{z}$ : angoli formati dall'asse $\xi$ rispettivamente con gli assi $x, y, z$ (Fig. 4).

a) Richiami sulla teoria del magnetometro con l'approssimazione del moto uniforme.

Il segnale ottenuto raggiunge il valore massimo, quando il centro del campione si trova ad una distanza $y$ dalla posizione di equilibrio dello stesso ordine di grandezza del raggrio $r$ della bobina. Ciò porta di conseguenza che per valori di $y$ superiori a quattro-cinque volte $r$, il segnale è praticamente nullo. Quindi si può considerare il pendolo in moto solo in questo piccolo intervallo: ed in questo intervallo, se il rapporto $r / l$ è molto piccolo, il moto può essere approssinato ad un moto uniforme, lungo l'asse $y$, con velocità costante $\frac{d y}{d t}=l \alpha_{0} \omega$.

Questa approssimazione porta una notevolissima semplificazione, in quanto in questo intervallo le tre componenti $m_{x}, m_{y}$ e $m_{z}$ del mo- 
mento magnetico $\vec{m}$ possono considerarsi indipendenti dal tempo e precisamente, essendo $a \simeq 0$ :

$$
\begin{aligned}
& m_{x}=m_{\xi} \cos \nu_{x}+m_{\eta} \cos \mu_{x}+m_{\zeta} \cos \lambda_{x} \\
& m_{y}=m_{\xi} \cos v_{y}+m_{\eta} \cos \mu_{y}+m_{\zeta} \cos \lambda_{y} \\
& m_{z}=m_{\xi} \cos \nu_{z}+m_{\eta} \cos \mu_{z}+m_{\zeta} \cos \lambda_{z}
\end{aligned}
$$

dove:

$$
\begin{aligned}
& m_{\xi}=m \operatorname{sen} \psi \cos \chi \\
& m_{\eta_{\gamma}}=m \operatorname{sen} \psi \operatorname{sen} \chi \\
& m_{\zeta}=m \cos \psi .
\end{aligned}
$$

Si la quindi per la differenza dei campi in $C_{1}$ e in $C_{2}$ :

$$
H_{1, \varepsilon}-H_{2, z}=-\frac{6 h a m_{x}}{R^{2}}
$$

dove

$$
R=\left[a^{2}+y^{2}+h^{2}\right]^{1 / 2},
$$

e derivando rispetto al tempo la [2] e la [3] ed eliminando la $\frac{d R}{d t}$

$$
\frac{d}{d t}\left(\ddot{I}_{1, \tau}-H_{2, z}\right)=\frac{30 \operatorname{ham}_{x}}{R^{6}} \frac{d R}{d t}=\frac{30 \operatorname{ha} m_{x}}{n^{2}} \eta l \alpha_{0} \omega m_{x}
$$

Questa approssimazione consiste nel trascurare le potenze dell'angolo a superiori alla prima, infatti la [5] della citata nota con questa approssimazione diventa:

$$
\frac{d}{d t}\left(H_{1,2}-H_{2, z}\right)=\frac{e}{3,17 \cdot 10^{-2}}=\frac{30 h a \omega a_{0} u l}{R^{2}} m_{x}
$$

che coincide con la precedente [4].

b) Campione decentrato e magnetizzato uniformemente.

Si supponga ora un campione, di dimensioni molto piccole e nialgnetizzato uniformemente, posto nel magnetometro in posizione non perfettamente centrata, cosicchè le sue coordinate rispetto alla terna di riferimento $\Omega, \xi, \eta, \zeta$ siano

$$
\xi=\delta_{\xi}, \quad \eta=\delta_{\eta}, \quad \zeta=\delta_{\zeta}
$$


Le coordinate rispetto alla terna $0, x, y, z$ ad un generico istante $t$ saranno cosi, essendo $\delta_{y}$ il valore di $y$ all'istante zero, quando cioè $O$ coincide con $\Omega$ :

$$
X=\delta_{x} \quad Y=\delta_{y}+v t \quad Z=\delta_{z} .
$$

Le componenti verticali del campo $H$ generato da questo campione nei centri delle due bobine sono rispettivamente

$$
\begin{aligned}
& \ddot{H}_{1, z}=\frac{m_{z}}{R_{1}^{3}}-3 \bar{u} \frac{\left(\delta_{x}-a\right) m_{x}+\left(\delta_{y}+v t\right) m_{y}+\left(\delta_{z}-a\right) m_{z}}{R_{1}^{5}} \\
& H_{2, z}=\frac{m_{z}}{R_{n}^{3}}-3 a \frac{\left(\delta_{x}+a\right) m_{x}+\left(\delta_{y}+v t\right) m_{y}+\left(\delta_{z}-a\right) m_{z}}{R_{n}^{5}},
\end{aligned}
$$

dove:

$$
\begin{aligned}
& R_{1}=\left[\left(\delta_{x}-a\right)^{2}+\left(\delta_{y}+v t\right)^{2}+\left(\delta_{z}-a\right)^{2}\right]^{1 / 2} \\
& R_{2}=\left[\left(\delta_{x}+a\right)^{2}+\left(\delta_{y}+v t\right)^{2}+\left(\delta_{z}-a\right)^{2}\right]^{1 / 2}
\end{aligned}
$$

La differenza tra le componenti $H_{z}$ è così:

$$
\begin{gathered}
H_{1, z}-H_{2, z}=\left(\frac{1}{R_{1}^{3}}-\frac{1}{R_{2}^{3}}\right) m_{z}-3 a\left(\frac{1}{R_{1}^{5}}-\frac{1}{R_{2}^{5}}\right) \\
{\left[\delta_{x} m_{x}+\left(\delta_{y}+v t\right) m_{y}+\left(\partial_{z}-a\right) m_{z}\right]+\left(\frac{1}{R_{1}^{5}}+\frac{1}{R_{2}^{5}}\right) 3 a^{2} m_{x} .}
\end{gathered}
$$

Ma $R_{2}$ si può esprimere in funzione di $R_{1}$ e di $\delta_{\xi}$ con uno sviluppo in serie, arrestato al solo primo termine:

$$
R_{2}=R_{1}\left(1+\frac{2 a \delta_{2}}{R_{1}^{2}}\right),
$$

e sostituendo questa espressione in [7] si ha:

$$
\begin{gathered}
H_{1, z}-H_{2, z}=\frac{6 a}{R_{1}^{5}}\left\{\delta_{x} m_{z}-\right. \\
\left.-\left|\delta_{x} m_{x}+\left(\delta_{y}+v t\right) m_{y}+\left(\delta_{z}-a\right) m_{z}\right| \frac{5 a \delta_{x}}{R_{z}^{z}}+a m_{x}\left(1-\frac{5 a \delta_{x}}{R_{1}^{z}}\right)\right\},
\end{gathered}
$$

ed ordinando:

$$
\begin{aligned}
& H_{1, z}-H_{2, z}==\frac{6 a}{R_{1}^{5}}\left\{m_{x} a\left[1-5 \frac{a \delta_{x}}{R_{1}^{2}} 1-5\left(\frac{\left.\delta_{x}\right|^{2}}{R_{1}}\right)\right]-\right. \\
& \left.\left.-5 m_{\nu} a \frac{\delta_{-}}{\Pi_{i}^{z}}\left(\delta_{y}+v t\right)+m_{z} \delta_{x} \mid 1-\frac{5 a^{2}}{R_{1}^{2}}\left(\frac{\delta}{a}-1\right)\right]\right\},
\end{aligned}
$$


se si trascurano i termini di secondo grado in $\delta_{x}, \delta_{y}$ e $\delta_{x}$ si ha:

$$
H_{1, z}-H_{2, z}=\frac{6 a}{R_{1}^{5}}\left[m_{x} a+m_{2} \delta_{x}\left(1+\frac{\tilde{s} u^{2}}{R_{2}^{2}}\right)\right]-\frac{30 a^{2}}{R_{1}^{7}} \delta_{x} v t m_{y}
$$

e la f.e.m. ottenuta ai capi delle due bobine:

$$
\begin{gathered}
e=\varphi \frac{d\left(H_{1, z}-H_{2, z}\right)}{d t}=\varphi\left\{\left[-\frac{30 a}{R_{1}^{6}}\left(m_{x} a+m_{z} \delta_{x}\left(1+\frac{j a^{2}}{R^{9}}\right)\right)--\right.\right. \\
\left.\left.-\frac{6 a}{R_{1}^{5}} m_{z} \delta_{x} \frac{10 a^{2}}{\pi_{1}^{3}}+\frac{210}{R_{1}^{8}} a^{2} \delta_{x} v t m_{\nu}\right] \frac{d R_{1}}{d t}-\frac{30 a^{2} \delta_{x}}{R_{1}^{7}} m_{y} v\right\}
\end{gathered}
$$

dove $\varphi$ tiene conto della geometria delle due bobine. Dalla prima delle [6] si ha anche:

$$
\frac{d R_{1}}{d t}=\frac{\delta_{y}+v t}{R_{1}} v
$$

che sostituita nella precedente relazione [12] dà:

$$
e=-\varphi \frac{30 a^{2} v}{R_{2}^{7}-}\left\{m_{x}\left(\delta_{y}+v t\right)+m_{y} \delta_{x}+m_{z} \delta_{x} v t\left(1+\frac{\tau a^{2}}{R_{1}^{2}}\right)\right\}
$$

avendo trascurato il termine $7\left(\frac{\delta_{y}+v t}{R_{1}}\right)^{2}$ rispetto ad uno.

Questa f.e.m. è quindi composta di tre termini che ora esamineremo separatamente.

Il primo è:

$$
-30 \varphi a^{2} v m_{x} \frac{\delta_{y}+v t}{\pi_{1}}=-30 \varphi a^{2} v m_{x} \underset{R_{1}^{2}}{y},
$$

il secondo è:

$$
-30 \varphi a^{2} v m_{\nu} \frac{\partial_{x}}{n_{\perp}},
$$

ed il terzo infine è:

$$
-30 \varphi u^{2} v m_{z} \frac{\delta_{-}}{a}\left(1+\tau \frac{a^{2}}{R_{1}^{2}}\right) \frac{y}{R_{1}^{7}} .
$$

Il primo termine è del tipo della [4], ciò che significa un segnale avente l'andamento della $f_{1}$ riportata in Fig. 5, rappresentata da una 
funzione dispari rispetto all'istante $t=-\frac{\delta_{1}}{v}$ che assume il valore più grande in modulo negli istanti:

$$
t_{1}=\frac{1}{v}\left(\frac{R_{o}}{\sqrt{6}}-\delta_{\nu}\right) \text { e } t_{2}=\frac{1}{v}\left(2 \frac{R_{o}}{\sqrt{6}}-\delta_{1}\right)
$$

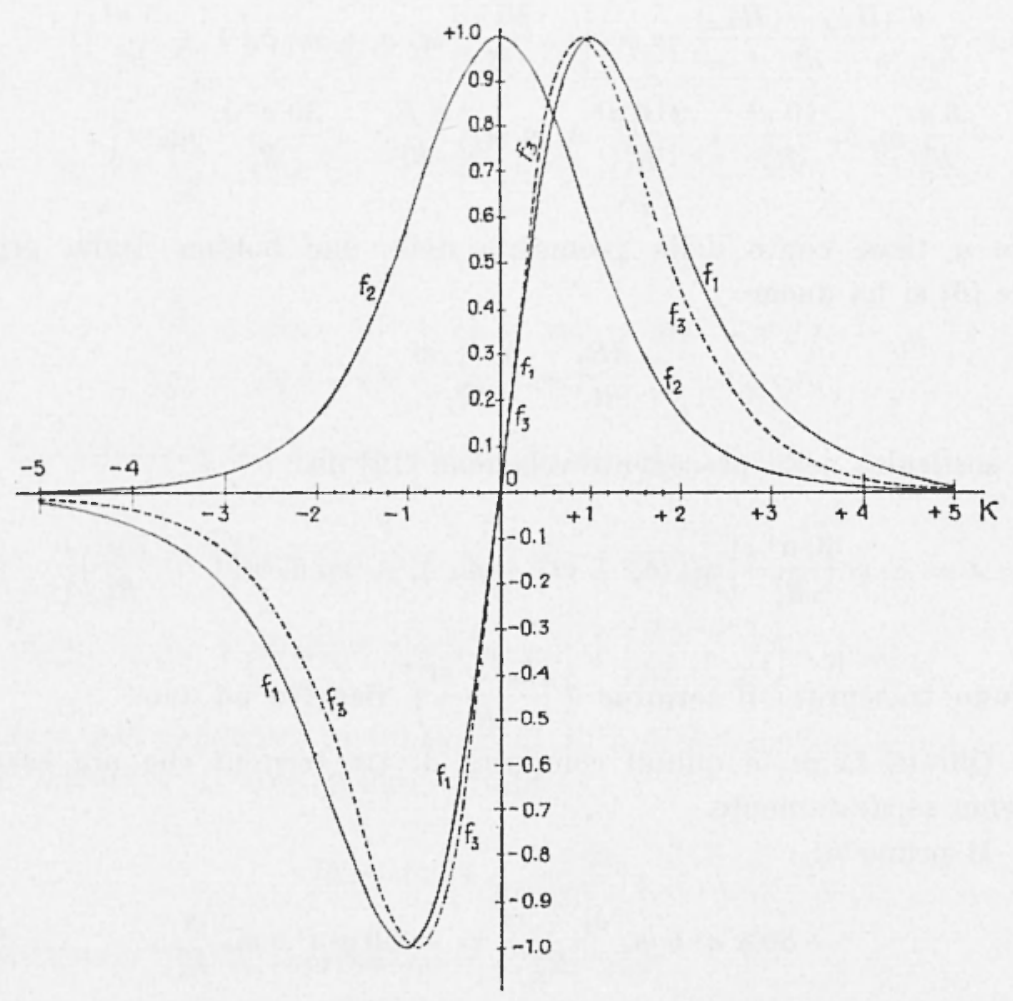

Fig. 5

dove:

$$
R_{0}=\left[\left(\delta_{x}-a\right)^{2}+\left(\dot{\delta}_{z}-a\right)^{2}\right]^{1 / 2}
$$

Infatti derivando rispetto a $y$ ed annullando la [14] si trova:

$$
y= \pm \frac{R_{0}}{\sqrt{6}}
$$


Il rapporto tra il valore di questo termine $e_{1}$ in un generico istante $t$ ed il suo valore massimo è dato da:

$$
\frac{e_{1}}{e_{1, M}}-\because \frac{\sqrt{6}}{R_{0}} \frac{(7 / 6)^{7 / 2}}{\left(1+\frac{y^{2}}{R_{0}^{2}}\right)^{7 / 2}}
$$

Si può ora esprimere là $y$ in unità $R_{0} / \sqrt{6}$, cioè in unità corrispondenti al valore di $y$ per cui la f.e.m. raggiunge il valor massimo ottenendo cosi, avendo posto $y=k R_{0} / \sqrt{6}$, la funzione $f_{1}$ della Fig. 5,

$$
f_{1}=\frac{e_{1}}{e_{\mathrm{r}, M}}=\tau^{7 / 2} \frac{k}{\left(6+l^{2}\right)^{7 / 2}} \text {. }
$$

Il secondo termine rappresenta una f.e.m. inversamente proporzionale a $R_{1}^{7}$ e ponendo $R_{1}^{2}=R_{0}^{2}+y^{2}$, si ha:

$$
-30 \varphi a^{2} v m_{\nu} \frac{\delta_{x}}{r_{?}^{7}}=-30 \varphi \frac{a^{2} v m_{y} \delta_{x}}{R_{0}^{7}} \cdot \frac{1}{\left[1+\left(\frac{y \prime}{R_{0}}\right)^{2}\right]^{7 / 2}},
$$

ed esprimendo la $y$ con la stessa unità di prima si ha:

$$
-30 \varphi \frac{a^{2} v m_{y} \delta_{x}}{R_{s}^{7}} \cdot\left(\frac{6}{6+k^{2}}\right)^{7 / 2}
$$

e quindi rappresenta un segnale dello stesso segno di $-m_{y}$ e che raggiunge il valore massimo per $k=0$ : l'andamento ̀̀ rappresentato dalla $f_{2}$ riportata nella Fig. 5, funzione pari rispetto a $y=0$, ottenuta facendo il rapporto tra il valore generico $e_{2}$ del secondo termine e il valore massimo da esso raggiunto:

$$
f_{2}=\frac{e_{2}}{e_{2, M}}=\left(\frac{6}{6+k^{2}}\right)^{7 / 2} \text {. }
$$

Il terzo termine infine è proporzionale a:

$$
\frac{y}{R_{1}^{2}}\left(1+\frac{7 a^{2}}{R_{1}^{2}}\right)=y \frac{y^{2}+9 a^{2}}{\left(y^{2}+2 a^{2}\right)^{9 / 2}}
$$

avendo posto $R_{0}^{2}=2 a^{2}$. Esso è rappresentato da una funzione dispari rispetto a $y=0$, dove essa si annulla. La derivata della [22] rispetto a $y$ annullata porta all'equazione:

$$
\frac{y^{3}}{a^{6}}+11 \frac{y^{2}}{a^{2}}-3=0
$$


le cui due radici reali sono $y= \pm 0.516 a$, a cui corrispondono i due valori di $k== \pm 0.893$.

Il valore della f.e.m. dovuto al terzo termine è:

$e_{3}=-30 \varphi a^{2} v m_{z} \frac{\partial_{x}}{a} y \frac{y^{2}+9 a^{2}}{n_{0}^{9}}=-30 \varphi a^{2} v m_{z} \frac{\delta_{x}}{a} \frac{6^{3}}{h_{0}^{6}} \frac{k^{2}+27}{\left(k^{2}+6\right)^{2 / 2}}[24]$

ed il suo valore massimo si ha per $k-0.893$ : facendo il rapporto $e_{3} / e_{3, M}$ si trova la $f_{3}$ riportata nella Fig. 5:

$$
f_{3}=\frac{e_{3}}{e_{3, M}}=29402 \frac{k\left(k^{2}+27\right)}{\left(k^{2}+6\right)^{2 / 2}} .
$$

I tre termini avranno quindi i valori:

$$
\begin{aligned}
& \left(-30 a^{2} v \varphi\right) m_{x} f_{1} p_{1} \\
& \left(-30 a^{2} v \varphi\right) m_{y} f_{2} p_{2} \\
& \left(-30 a^{2} v \varphi\right) m_{z} f_{3} p_{3}
\end{aligned}
$$

dove $p_{1} p_{2}$, e $p_{3}$ sono i valori massimi positivi assunti dalle rispettive funzioni, e cioc:

$$
p_{1}=\frac{0,238}{R_{0}^{6}} \quad, \quad p_{2}=\frac{\delta_{x}}{R_{0}^{7}} \quad, \quad p_{3}=1,36 \frac{\delta_{x}}{R_{0}^{7}} .
$$

Ise $f_{1}, f_{2}$ e $f_{3}$ sono espresse tutte in funzione del parametro $k$, che ci indica la posizione del pendolo per la quale il primo termine assume il valore massimo: si è preso questo parametro come riferimento unico per tutte le funzioni, in quanto esso si riferisce al termine relativo al campione centrato.

Da un esame sommario delle tre curre $f_{1}, f_{2}$ e $f_{3}$ appare che le due funzioni $f_{1}$ e $f_{3}$ sono pressochè uguali, specie nel tratto compreso tra $k=-1$ e $k=+1$; ciò porta di conseguenza che, in una prima approssimazione, esse si possono considerare uguali. Con questa posizione i tre termini che compaiono nella [13] si possono riunire in due soli, ottenendo rosì:

$$
e=\left(-30 \varphi a^{2} v\right)\left\{\left(m_{x} p_{1}+m_{z} p_{3}\right) f_{1}+m_{y} p_{2} f_{2}\right\} .
$$

Il primo termine è rappresentato da una funzione dispari ed il secondo da una funzione pari.

Allo scopo di studiare la forma del segnale si può esprimere la [28] nel seguente modo, introducendo un coefficiente $\varrho$ che rappresenta il rapporto tra il coefficiente del secondo termine e quello del primo:

$$
c=\left(-30 \varphi a^{2} v\right)\left(0,238 m_{x}+1,36 \frac{\delta_{x}}{R_{0}} m_{z}\right)\left(f_{1}+o f_{2}\right) \text {. }
$$


Nella Fig. 6 ho riportato la forma del segnale per diversi valori di $\varrho$ e ciò̀ per $\varrho=0,1,5, \infty$. Si vede subito che al variare di $\varrho$, ciò che si può ottenere con una semplice rotazione del campione, il segnale

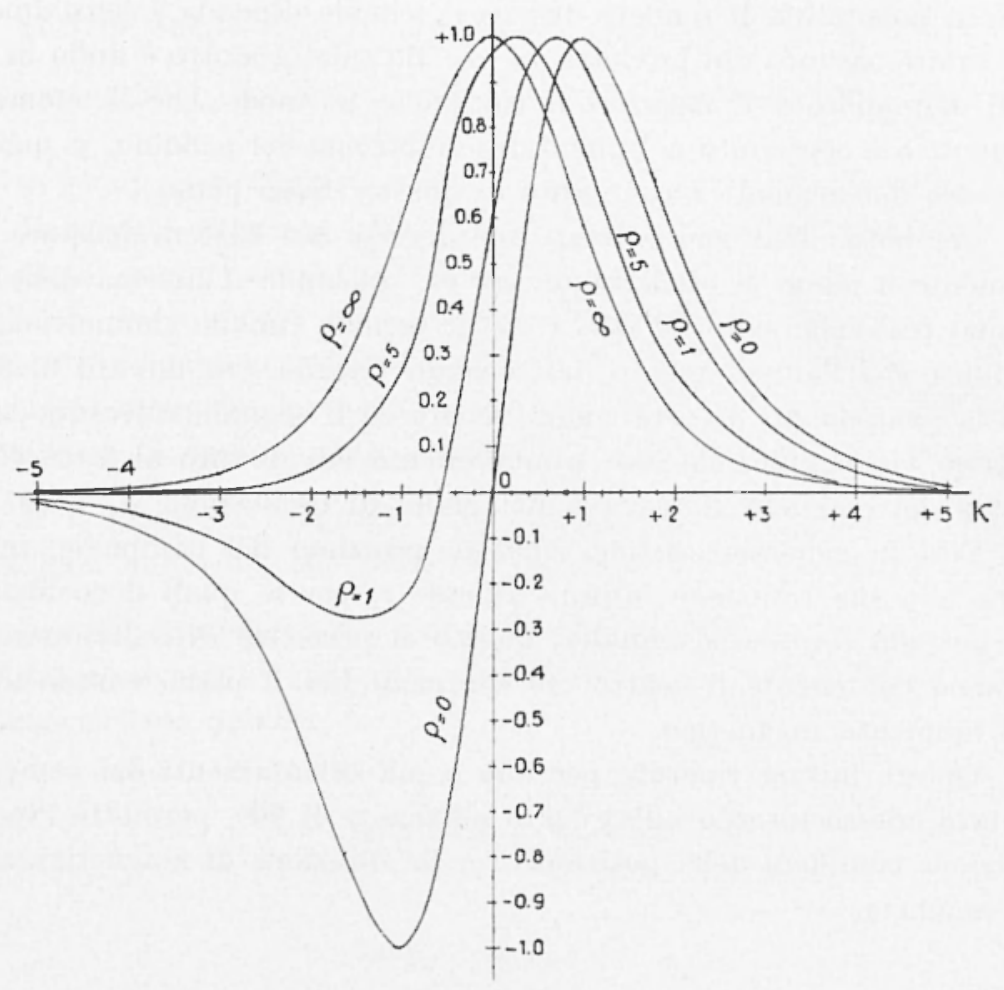

Fig. 6

cambia completamente di aspetto: da segnale simmetrico pari per $\varrho=\infty$, ciò che significa anche $0,238 m_{x}=-1,36 \frac{\delta_{z}}{F_{0}} m_{z}$, esso diventa asimmetrico per gli altri valori di $\underline{o}$ diversi da zero ed infine diventa simmetrico e dispari per $\varrho=0$, ciò che significa che il prodotto $m_{y} \cdot \delta_{x}$ è nullo.

Un magnetino decentrato contribuisce così al segnale per il termine $f_{1}$ (dispari) con un contributo proporzionale alla somma:

$$
0,238 m_{x}+1,36 \frac{\delta_{x}}{l_{0}} m_{x},
$$


e per il termine $f_{2}$ (pari) con un contributo proporzionale al prodotto

$$
m_{y} \frac{\delta_{x}}{R_{0}}
$$

Civè la possibilità di rendere dispari il segnale dipende essenzialmente dal valore assunto dal prodotto $m_{y} \cdot \delta_{x}$. Ma tale prodotto è nullo in due casi: o quando si è disposto il campione in modo che il momento magnetico è contenuto nel piano di oscillazione del pendolo, o quando il centro del magnete è contenuto in questo stesso piano.

Per poter distinguere questi due diversi casi basterà spostare leggermente il piano di oscillazione, per es. traslando il sistema delle due bobine parallelamente all'asse $x$. Se il segnale rimane simmetrico, ciò significa che l'annullamento del secondo termine era dovuto al fatto che la componente $m_{y}$ era nulla; se invece il segnale ridiventa asimmetrico, ciò significa che tale annullamento era dovuto al fatto che il centro del magnete si trovava nel piano di oscillazione $\delta_{x}=0$.

Cosi in generale esistono quattro posizioni del campione, in seguito alla sua rotazione intorno all'asse $z$, per le quali il coefficiente del secondo termine si annulla; questo ci permette di individuare sia il piano contenente il centro del campione sia il piano contenente il suo momento magnetico.

Queste misure ripetute per due o più orientamenti del campione ruotato adesso intorno all'asse $x$ o all'asse $y$ di $90^{\circ}$, permette l'individuazione completa della posizione e della direzione di magnetizzazione del magnete.

\section{c) Schematizzazione della disomogeneità di magnetizzazione.}

Un campione comunque magnetizzato puó essere ritenuto come un insieme di magnetini elementari magnetizzati in generiche direzioni non necessariamente parallele e con momenti magnetici di valore diverso. Pertanto i due termini del segnale complessivo saranno proporzionali per la $f_{1}$ a:

$$
0,238 \int_{v} I_{x} d v+\frac{1,36}{R_{0}} \int_{v} I_{z} x d v .
$$

e per la $f_{2}$ a:

$$
\frac{1}{R_{0}} \int_{v} I_{y} x d v
$$


dove $I_{x}, I_{y}, I_{z}$ sono le componenti del vettore intensità di magnetizzazione lungo gli assi $x, y, z, d v$ è l'elemento generico di volume del campione in esame ed $x$ è la sua distanza dal piano $y z$ : l'integrale va esteso a tutto il volume $v$ del campione.

$\mathrm{Si}$ vede subito che se il campione è magnetizzato uniformemente i due integrali $\int_{v} I_{z} x d v$ e $\int_{v} I_{y} x d v$ sono entrambi nulli, solo se il centro geometrico $\Omega$ del campione si trova nel piano di oscillazione.

Il nostro campione potrà essere perciò schematizzato come un campione magnetizzato uniformemente in tutto il suo volume, al quale sia sovrapposto un magnete elementare decentrato e con un momento magnetico non necessariamente parallelo al primo. L'effetto di questo magnete decentrato, che chiamerò magnete equivalente di disomogeneità sarà appunto quello di cambiare la forma del segnale. Sia $\vec{M}$ il momento magnetico del campione, dovuto alla sua magnetizzazione uniforme, che possiamo supporre applicato al centro geometrico del campione; sia inoltre $\vec{m}$ il momento magnetico del magnetino equivalente supposto decentrato di un tratto $\delta$. In questo schema avremo quindi:

$$
\begin{aligned}
& M_{x}+m_{x}=\int_{v} I_{x} d v \\
& m_{\nu} \delta_{x}=\int_{v}^{2} I_{y} x d v \\
& m_{z} \delta_{x}=\int_{v}^{v} I_{z} x d v
\end{aligned}
$$

Il segnale avrà allora l'espressione, in base alla [29]:

$$
e=\left(-30 \varphi a^{2} v\right)\left[0,238\left(M_{x}+m_{x}\right)+1,36 \frac{\delta_{x}}{l_{0}} m_{2}\right]\left(f_{1}+\varrho f_{2}\right),
$$

dove $\varrho$ è espresso dal rapporto:

$$
\varrho=\frac{m_{y} \frac{\delta_{x}}{R_{0}}}{0,238\left(M_{x}+m_{x}\right)+1,36 \frac{\stackrel{s}{\mathfrak{v}_{x}}}{R_{0}} m_{z}} .
$$


Il valore massimo raggriunto da questo rapporto potrà essere preso come indice della disomogeneità di magnetizzazione del campione in esitme (3).

Orbene so nella rotazione del campione intorno all'asse $z$ si osserva che il massimo positivo e quello negativo non sono esattamente eguali, riò denuncia l'esistenza della disomogeneità di magnetizzazione del campione. Per avere un'idea di tale disomogeneità si può fare subito il calcolo per valori di $\varrho$ molto piecoli. Se si indica con $\beta$ il rapporto tra il valore massimo del segnale positivo e quello massimo del segnale negativo (in modulo) si trova che tra po e o definito dalla [31] esiste questa relazione approssimata, valevole per $\varrho$ prossima a 0 :

$$
\beta=1+1,17 \varrho \text {. }
$$

Cosi se si impone la condizione per es. ehe il campione non deve presentare una disomogeneità maggiore del $10 \%$, si dovianno scartare tutti i campioni che presentano, in un qualsiasi orientamento, almeno un valore di $\beta$ maggiore di 1,12 .

Isa caratteristica principale di questo nuovo tipo di magnetometro è, oltre alla sua sensibilità nella determinazione della direzione della intensità di magnetizzazione ${ }^{(1)}$, la possibilità di individuare con un esame abbastanza rapido, quei campioni, i quali, per una qualsiasi causa, presentano una eccessiva disomogeneità di magnetizzazione. Questo tipo di esanre è necessario in quanto i campioni utilizzabili per le misure paleomagnetiche, devono presentare una certa uniformità di magnetizzazione. Infatti la direzione della intensita di magnetizzazione rilevata con un qualunque magnetometro, nel caso di magnetizzazione non uniforme, non ha generalmente una relazione immediata con la direzione della magnetizzazione del campione all'epoca della formazione della roceia( $\left.{ }^{4}\right)$.

\section{BIBIJOGRAFIA}

(') Cratidea R., Un nuovo tipo di magnetometro per le misure magnetiche sulle roce. "Annali di Geofisica", XIX, 4, (1966).

(2) Nagata T., Maynetic Properties of Rocks and Minerals, "Handbuch der Pliysik ", Bd. XIIX, 1, Springer-Verlag, (1966). Colfinson D). W., Creer K. M., Runcorn S. K.. Methods in Paleomagnetism, Elsevier, 1967.

Runcorn S. K., Magnetization of Rocks, "Handbuch der Physik", Bd. XLVIl, Springer-Verlag, (1956).

(3) Irwing E., Molyneux J., Runcorn S. K., Geophys. J., 10, p. 451, (1966).

(4) Nagata T.. Rock Magnetism, Maruzen, $196 \mathrm{l}$. 